\title{
Relação entre crises epilépticas e lesões no complexo maxilofacial:
}

\section{Relationship between epileptic seizures and orofacial injuries}

\author{
MAITE BERTOTTI ${ }^{1}$ \\ BRUNA LUIZA ROIM VAROTTO ${ }^{2}$ \\ Rita de CÁssia d'OtTAVIANo Nápole ${ }^{3}$ \\ REYNALDO ANTEQUERA ${ }^{4}$
}

\section{RESUMO}

Objetivo: Este estudo tem como objetivo realizar uma revisão da literatura sobre a relação entre crises epilépticas e lesões no complexo maxilofacial.

Métodos: Para adequada análise, desenvolveu-se uma estratégia de revisão de literatura na base de dados PubMed, Cochrane e Scielo, abrangendo publicações de 1993 a 2017. Revisões de literatura e relatos de caso clínico foram definidos como critérios de exclusão

Resultados: Foram selecionados 15 artigos, os quais incluem análise de coorte, caso controle e estudos transversais. Verificou-se escassez de estudos clínicos referentes ao assunto. Apesar da dificuldade de generalizar os resultados dos diferentes estudos selecionados, verificou-se uma associação direta entre as crises epilépticas e as lesões de tecidos moles orais, especialmente em relação à língua, lábios e mucosa jugal. Estruturas dentárias também são amplamente afetadas, e, em uma menor incidência, encontram-se as fraturas de mandíbula. Além disso, atitudes como inserção de objetos entre os dentes durante as crises comprometem diretamente o aparecimento de lesões. Lesões bucomaxilofaciais são capazes de causar prejuízos funcionais e estéticos, além de sintomatologia dolorosa.

Conclusão: O cirurgião-dentista tem papel fundamental não apenas no manejo, mas também na prevenção dessas lesões. Ampliar o acesso à informação e oferecer medidas preventivas podem ter impacto direto na redução da prevalência das lesões associadas às crises epilépticas.

Palavras-chave: Convulsões. Epilepsia. Traumatismos faciais. Traumatismos dentários.

\footnotetext{
ABSTRACT

Objective: This study aims to conduct a literature review regarding the relationship between seizures and orofacial injuries.

Methods: It was developed a strategy of review of literature in PubMed, Cochrane and Scielo databases from 1993 to 2017. After analyzing the titles and the abstracts and reading the full articles, the appropriated studies were selected. Literature reviews and case reports were defined as exclusion criteria.
}

\footnotetext{
Residente em Cirurgia e Traumatologia Buco-Maxilo-Facial no Hospital das Clínicas da Faculdade de Medicina da Universidade de São Paulo, Brasil

2 Mestre em Ciências da Saúde pelo Hospital das Clínicas da Faculdade de Medicina da Universidade de São Paulo, Brasil

3 Cirurgiã Dentista Assistente da Equipe Odontológica do Instituto de Psiquiatria, Hospital das Clínicas da Faculdade de Medicina da Universidade de São Paulo, Brasil

4 Cirurgião Dentista Supervisor da Equipe Odontológica do Instituto de Psiquiatria, Hospital das Clínicas da Faculdade de Medicina da Universidade de São Paulo, Brasil
} 
Results: It was selected 15 studies, which include cohort analysis, case control, cross-sectional studies. There was also a lack of clinical studies on the subject. Despite the difficulty of generalizing the results of the different selected studies, there is a direct association between epileptic seizures and oral soft tissues injuries, especially affecting the tongue, lips and cheek. Dental structures are also widely affected, and, at a lower incidence, jaw fractures can be detected. In addition, some cultural beliefs and attitudes, such as the insertion of objects between the teeth, are directly associated with the appearance of lesions after epileptic seizures. Orofacial lesions may cause functional and aesthetic damage, as well as painful symptomatology.

Conclusion: Dentists play a fundamental role not only in the management of these lesions, but also in their prevention. Increasing access to information and providing preventive measures can have a direct impact on reducing the prevalence of seizure-related injuries.

Keywords: Seizures. Epilepsy. Facial injuries. Tooth injuries.

\section{INTRODUÇÃO}

Crises epilépticas são perturbações involuntáriasparoxísticasdafunçãocerebralque podem se manifestar como comprometimento de consciência, atividade motora anormal, anormalidades comportamentais, distúrbios sensoriais ou disfunção autonômica. ${ }^{1}$ Durante uma crise, ocorre perda temporária do controle da tonicidade muscular, tendo associação direta com traumatismo em face e na cavidade oral. ${ }^{2}$ Desse modo, lesões do complexo bucomaxilofacial podem ser observadas, principalmente com crises generalizadas. ${ }^{3,4}$ Essas lesões podem causar morbidades associadas a prejuízo estético, quadros infecciosos, dor orofacial, dificuldade mastigatória e mal oclusão. ${ }^{1}$

O objetivo deste trabalho foi verificar a relação entre crises epilépticas e traumatismos do complexo bucomaxilofacial.

\section{MÉtodos}

Realizou-se uma revisão da literatura nas bases de dados PubMed, Cochrane e Scielo, utilizando a combinação dos seguintes termos: "Orofacial trauma" ou "Orofacial injury" e "Seizures" ou "Epilepsy". A busca foi realizada em abril de 2018.

Os critérios de inclusão foram artigos publicados no idioma inglês, a partir de 1993, do tipo coorte, caso controle e estudos transversais. Os critérios de exclusão foram trabalhos de revisões de literatura e relatos de casos, artigos em outros idiomas que não o inglês, ouartigosanterioresà 1993. Inicialmente, analisaram-se títulos e resumos relacionados com as palavras-chave; após, prosseguiu-se com a leitura dos estudos na íntegra. Para facilitar a análise dos artigos selecionados, fezse o registro das informações por meio de uma tabela, subdividida em autores, local, amostra, metodologia e resultados.

\section{Resultados}

Foram incluídos 15 artigos científicos envolvendo crises epilépticas e traumatismos no complexo bucomaxilofacial, entre os anos de 1993 e 2017. Não foram encontrados artigos sobre o tema publicados entre janeiro e abril de 2018. Os artigos estão dispostos na Tabela 1 e 2, de acordo com o tipo de estudo.

A prática cultural de interpor objetos nas arcadas dentárias durante as convulsões desempenha um papel importante na ocorrência das lesões. Adicionalmente, a maioria das lesões foi relacionada às convulsões generalizadas. , $, 6,7^{-}$

Entre os fatores de risco se incluem convulsões tônico-clônicas generalizadas, com, pelo menos, 12 crises epilépticas por ano e a ocorrência de convulsões diurnas, compatível com a quantidade de atividades nesse período. ${ }^{3,8}$ 
Tabela 1 - Estudos prospectivos

\begin{tabular}{|c|c|c|c|}
\hline Autores & Amostra & Metodologia & Resultados \\
\hline $\begin{array}{l}\text { Nakken e Lossius } \\
(1993)^{6}\end{array}$ & $\begin{array}{l}\text { Epilepsia severa: } \\
\mathrm{N}=62\end{array}$ & $\begin{array}{l}\text { Coorte (13 } \\
\text { meses) }\end{array}$ & $\begin{array}{l}\text { Risco de lesão relacionado às crises: } 1,2 \% \text {. } \\
\text { Lesões frequentes: tecidos moles. Crises } \\
\text { generalizadas. }\end{array}$ \\
\hline $\begin{array}{l}\text { Kirby e Sadler } \\
(1995)^{17}\end{array}$ & Epilépticos N=560 & $\begin{array}{l}\text { Estudo } \\
\text { transversal ( } 1 \\
\text { ano) }\end{array}$ & $\begin{array}{l}\text { Traumas ou óbitos: } n=84(15 \%) \text {. } \\
\text { Lesões em tecido mole (contusões e } \\
\text { lacerações). }\end{array}$ \\
\hline $\begin{array}{l}\text { Broek e Beghi } \\
(2004)^{13}\end{array}$ & $\begin{array}{l}\text { 1) Epilépticos n=951 } \\
\text { 2) } G C n=904\end{array}$ & $\begin{array}{l}\text { Caso-controle } \\
(1-2 \text { anos })\end{array}$ & $\begin{array}{l}\text { Acidentes: epilépticos n=199 ( } 21 \%), G C \text { n=123 } \\
(14 \%) \text {. Tipos de lesões: concussão, abrasão e } \\
\text { FCC. }\end{array}$ \\
\hline $\begin{array}{l}\text { Ndukwe et al. } \\
(2007)^{12}\end{array}$ & $\begin{array}{l}\text { Convulsões febris em } \\
\text { crianças } \mathrm{N}=75\end{array}$ & $\begin{array}{l}\text { Estudo } \\
\text { transversal }\end{array}$ & $\begin{array}{l}\text { Lesões bucomaxilofaciais n=27 (36\%). Causa: } \\
\text { interposição de colher entre as arcadas } \\
(96,3 \%) \text {. }\end{array}$ \\
\hline $\begin{array}{l}\text { Adewole et al. } \\
(2011)^{5}\end{array}$ & Epilépticos N=138 & $\begin{array}{l}\text { Coorte }(2 \\
\text { anos) }\end{array}$ & $\begin{array}{l}\text { Lesões bucomaxilofaciais: } \mathrm{n}=87(63 \%) \text { - } \\
\text { tecidos moles (língua). Fatores de risco: CTCG } \\
\text { e interposição de objetos entre as arcadas. }\end{array}$ \\
\hline $\begin{array}{l}\text { Adeyemo et al. } \\
(2011)^{9}\end{array}$ & $\begin{array}{l}\mathrm{N}=257 \text { (emergência } \\
\text { pediátrica) }\end{array}$ & Coorte (1 ano) & $\begin{array}{l}\text { Lesões bucomaxilofaciais n=13 (5,1\%). } \\
\text { Tecidos moles: } n=12(92,3 \%) \text {. Fator de risco: } \\
\text { interposição de objeto entre as arcadas. }\end{array}$ \\
\hline $\begin{array}{l}\text { Gawlak et al. } \\
(2017)^{10}\end{array}$ & Epilépticos $\mathrm{N}=100$ & $\begin{array}{l}\text { Estudo } \\
\text { transversal }(1 \\
\text { ano) }\end{array}$ & $\begin{array}{l}\text { Lesão bucal: } 52,4 \% \text {. Tecidos moles: língua, } \\
\text { lábios e mucosa jugal. Dentes: trincas, fraturas } \\
\text { e avulsão. }\end{array}$ \\
\hline
\end{tabular}

Legenda: GC = grupo controle, FCC = ferimento corto-contuso, CTCG = convulsões tônico-clônicas generalizadas.

Tabela 2 - Estudos Retrospectivos

\begin{tabular}{|c|c|c|c|}
\hline Autores & Amostra & Metodologia & Resultados \\
\hline $\begin{array}{l}\text { Ogunbodede et al. } \\
\qquad(1998)^{16}\end{array}$ & $N=56$ & $\begin{array}{l}\text { Estudo } \\
\text { transversal (6 } \\
\text { meses) }\end{array}$ & $\begin{array}{l}\text { Traumatismos dos dentes anteriores: } n=26 \\
\qquad(46,4 \%)\end{array}$ \\
\hline Thomas et al. (2009) ${ }^{7}$ & $N=1673$ & $\begin{array}{l}\text { Estudo } \\
\text { transversal (5 } \\
\text { anos) }\end{array}$ & $\begin{array}{l}\text { Lesões dentárias } n=14 \text { : incisivos } n=10 \text {. } \\
\text { Tipo de epilepsia: mioclônica juvenil }(E M J) n=8\end{array}$ \\
\hline $\begin{array}{l}\text { Tiamkao et al. } \\
(2009)^{21}\end{array}$ & $N=300$ & $\begin{array}{c}\text { Estudo } \\
\text { transversal }\end{array}$ & $\begin{array}{l}\text { Fatores de risco: CTCG; pelo menos } 12 \text { crises, } \\
\text { ano; período diurno. }\end{array}$ \\
\hline Costa et al. $(2011)^{15}$ & $\begin{array}{l}\mathrm{N}=109 \text { (epilepsia } \\
\text { refratária + traumas) }\end{array}$ & $\begin{array}{c}\text { Estudo } \\
\text { transversal }\end{array}$ & $\begin{array}{l}\text { Fraturas dentárias coronárias }(n=42) \text {, avulsão } \\
(n=8) \text {, fraturas mandibulares }(n=8) .\end{array}$ \\
\hline $\begin{array}{l}\text { Gerreth K e Gerreth P } \\
(2014)^{14}\end{array}$ & $\begin{array}{c}N=126: \\
\text { 1) } n=63 ; 2) G C: n=63\end{array}$ & Coorte & $\begin{array}{l}\text { Fratura de coroa em dentes permanentes: } \\
n=10(15,9 \%), 13 \text { incisivos. GC: fratura dentária } \\
(6,4 \%) .\end{array}$ \\
\hline Ghafoor et al. $(2014)^{11}$ & $\begin{array}{l}\mathrm{N}=150 \text { (crianças em } \\
\text { tratamento por } 1 \text { ano) }\end{array}$ & $\begin{array}{c}\text { Estudo } \\
\text { transversal }\end{array}$ & $\begin{array}{l}\text { Traumas mais prevalentes: tecidos moles } \\
\text { (língua e mucosa jugal), tecidos duros (fratura } \\
\text { dentária). }\end{array}$ \\
\hline
\end{tabular}

Legenda: $\mathrm{GC}$ = grupo controle, FCC = ferimento corto-contuso, CTCG = convulsões tônico-clônicas generalizadas.

FOL • Faculdade de Odontologia de Lins/Unimep • 28(2) 49-55 • jul.-dez. 2018 
Os tecidos moles são comumente acometidos, especialmente língua, lábios e mucosa jugal.5,6,9,10,11,12. Em relação aos tipos de lesão, destacam-se lacerações, contusões e abrasões. ${ }^{13}$

No que concerne às estruturas dentárias, há maior prevalência de fraturas e trincas nos incisivos e caninos devido à localização desses dentes, estando mais suscetíveis a traumas. ${ }^{5,14} \mathrm{~A}$ diferença entre a prevalência de fraturas entre os estudos pode ser explicada pela diferença no desenho do estudo e pelo contexto social dos participantes, visto que a prática de interpor objetos entre as arcadas tem relação direta com as lesões orofaciais. ${ }^{15,16}$ Além disso, na infância as avulsões são mais frequentes devido à fragilidade do tecido ósseo, enquanto nos adultos há maior ocorrência de fraturas dentárias. ${ }^{4}$

A maioria das lesões reportadas foi de pequena intensidade; no entanto, Kirby e Sadler notificaram sete pacientes em óbito durante as convulsões. ${ }^{17}$ No estudo de Nakken e Lossius confirmou-se a possibilidade de lesões graves e, até mesmo, com risco de vida. ${ }^{6}$

Em relação ao manejo, recomenda-se o uso de capacetes de segurança ou protetores bucais, especialmente nos casos de lesões recorrentes. ${ }^{18}$ Protetores bucais proporcionam estabilização e absorção de energia, prevenindo traumas e reduzindo o potencial de energia transferida para a articulação temporomandibular e base do crânio. Tais protetores podem diminuir significativamente as lesões especialmente nos pacientes capazes de prever uma crise epiléptica devido aos sintomas prodrômicos ou aura, os quais constituem em torno de $42 \%$ dos pacientes. ${ }^{10}$

\section{Dıscussão}

O presente estudo justifica-se pela necessidade de pesquisas confiáveis que evidenciem as variáveis relacionadas com a associação de crises epilépticas e traumas bucomaxilofaciais. A evidência existente sobre o assunto corrente, apresenta-se escassa em relação à amostra. Além disso, há limitação de estudos de caso-controle e ensaios clínicos.

Os principais preditores associados a essas lesões são gravidade, frequência e duração das crises. 3,14,19,20,21 No entanto, apesar de a frequência estar diretamente relacionada com a quantidade de lesões, Adewole et al. não encontraram significância estatística entre tal relação. 5,6

Há alguns resultados conflitantes em relação ao risco geral dos acidentes e da proporção de risco atribuível à epilepsia. Tais contradições podem ser explicadas por diferenças na amostra e na metodologia empregada. ${ }^{13}$ As diferenças entre os achados também podem ser explicadas pelos diferentes níveis de educação dos participantes, tendo relação direta com acesso à informação. ${ }^{12}$ Outra razão para a diferença de resultados entre os estudos pode estar diretamente associada com a gravidade da epilepsia dos pacientes, visto que a associação de fármacos sugere falha no controle com a monoterapia.

De acordo com os dados supracitados, pode-se observar a dificuldade de generalização dos resultados em nível populacional, uma vez que os achados são diretamente dependentes de algumas variáveis, como classificação da crise epiléptica, controle farmacológico, tamanho da amostra disponível, faixa etária associada, condição socioeconômica e acesso à informação. Portanto, o desafio de se manter um parâmetro específico da condição e das lesões que podem estar associadas à mesma demonstra a importância da entrevista dialogada desses pacientes, prevendo uma anamnese detalhada com foco no histórico médico e nas características demográficas desses pacientes. Da mesma forma, um exame clínico minucioso pode servir como 
método comparativo pós-crise epiléptica, sendo a sua documentação fundamental nesses casos.

\section{Considerações Finais}

Uma vez que há maior risco de acidentes em pacientes com epilepsia ativa e generalizada, sugere-se que as convulsões não controladas podem representar ameaça direta para lesões orofaciais, sendo indispensável o correto acompanhamento desses pacientes. Os resultados desta revisão de literatura relacionam maior prevalência de lacerações, abrasões e contusões de tecidos moles, especialmente em lábios, língua e mucosa jugal. Em relação aos tecidos duros, houve maior associação da condição com fraturas dentárias.

Lesões bucomaxilofaciais podem causar morbidade significativa, incluindo prejuízo estético e funcional, como diminuição da eficiência mastigatória, mal oclusão e sintomatologia dolorosa. Assim, medidas preventivas são tão importantes quanto o manejo apropriado dessas lesões, as quais incluem abordagens educativas e esclarecimentos de condutas deletérias de cunho cultural. O manejo profilático inclui a utilização de protetores bucais e a expansão do atendimento odontológico nesses pacientes.

Portanto, o cirurgião-dentista tem papel fundamental em manter a qualidade da função mastigatória, evitar prejuízos estéticos, reduzira sintomatologia dolorosa e manter a estabilidade da qualidade de vida desses pacientes. Tal entendimento pode ser um diferencial na formação dos cirurgiões-dentistas, uma vez que traumatismos orofaciais acometem grande parte dos pacientes epilépticos.

\section{Contribuições:}

MB trabalhou na concepção, delineamento, análise e interpretação dos estudos incluídos, bem como, redação do trabalho.

BLRV coordenou, supervisionou e revisou criticamente o trabalho.

RA e RCON participaram da revisão crítica do trabalho e aprovação da versão final a ser publicada.

Todos os autores aprovaram o manuscrito final como apresentado e concordam em prestar contas de todos os aspectos do trabalho.

Conflito de Interesse: Os autores declaram que não há conflitos de interesse.

Fonte de Financiamento: Nenhum financiamento foi garantido para este estudo.

Divulgação Financeira: Os autores não têm relações financeiras relevantes a divulgar.

\section{REFERÊNCIAS}

1. Haslam RHA, Seizures in childhood. In: Nelson WE, Behrman RE, Kliegman RM, Nelson Textbook of Pediatrics, 15th ed., Philadelphia: Saunders, 1996, p. 1.686-99.

2. Aragon CE, Burneo JG, Helman J. Occult maxillofacial trauma in epilepsy. J Contemp Dent Pract. 2001, 2 (4): 26-32.

ht t p s: // ww w.ncbi.n Im.nih.gov/ pubmed/12167918

3. Mollaoğlu M, Bolayir E. Injuries in Patients with Epilepsy and Some Factors Associated with Injury. Noro Psikyatr Ars. 2013, 50 (3): 269-73. https://www.ncbi.nIm.nih.gov/pmc/articles/ PMC5363446/pdf/npa-50-3-269.pdf

4. Nonato ER, Borges MA. Oral and maxillofacial trauma in patients with epilepsy: prospective study based on an outpatient population. Arq Neuro-Psiquiatr. 2011, 69 (3): 491-5. http://www.scielo.br/pdf/anp/v69n3/a16v69n3. pdf 
5. Adewole RA, Ojini FI, Akinwande JA, Danesi MA. Oro-Dental and Maxillofacial Trauma in Epilepsy at a Tertiary Hospital in Lagos. West Afr J Med. 2011, 30 (2): 114-7.

ht t p s: // w w w.ncbi.n I m.nih.gov/ pubmed/21984459

6. Nakken KO, Lossius R. Seizure-related injuries in multihandicapped patients with therapy-resistant epilepsy. Epilepsia. 1993, 34 (5): 836-40.

https://onlinelibrary.wiley.com/doi/ abs/10.1111/j.1528-1157.1993.tb02099.x

7. Thomas RH, Higgins S, Fuller GN. Dental injuries during seizures associated with juvenile myoclonic epilepsy. J Neurol Neurosurg Psychiatry. 2009, 80 (1): 91-93.

https://jnnp.bmj.com/content/80/1/91.long

8. Martin BS. Traumatic intrusion of maxillary permanent incisors into the nasal cavity associated with a seizure disorder: report of a case. Dent Traumatol. 2003, 19 (5): 286-8.

https://onlinelibrary.wiley.com/doi/abs/10.1034/ j.1600-9657.2003.00170.x

9. Adeyemo WL, Fajolu IB, Temiye EO, Adeyemi MO, Adepoju AA. Orofacial and dental injuries associated with seizures in paediatric patients in Lagos University Teaching Hospital. Int J Pediatr Otorhinolaryngol. 2011, 75 (5): 670-2. https://www.sciencedirect.com/science/article/ pii/S0165587611000711?via\%3Dihub

10. Gawlak D., Łuniewska J., Stojak W., Hovhannisyan A., Strózynska A., Manka-Malara $\mathrm{K}$. et al. The prevalence of orodental trauma during epileptic seizures in terms of dental treatment - Survey study. Neurol Neurochir Pol. 2017, 51 ( 5): 361-5.

https://www.sciencedirect.com/science/article/ pii/S0028384317302232?via\%3Dihub

11. Ghafoor PA, Rafeeq M., Dubey A. Assessment of oral side effects of antiepileptic drugs and traumatic oro-facial injuries encountered in epileptic children. J Int Oral Health. 2014, 6 (2): 126-8. https://www.ncbi.nlm.nih.gov/pmc/articles/ PMC4037794/

12. Ndukwe KC, Folayan MO, Ugboko VI, Elusiyan JBE, Laja OO. Orofacial injuries associated with prehospital management of febrile convulsion in Nigerian children. Dent Traumatol. 2007. 23 (2): $72-5$.

https://onlinelibrary.wiley.com/doi/abs/10.1111/ j.1600-9657.2005.00411.x

13. Broek M., Beghi E. Accidents in patients with epilepsy: types, circumstances, and complications: an european cohort study. Epilepsia. 2004, 45 (6): 667-72.

https://onlinelibrary.wiley.com/doi/abs/10.1111/ j.0013-9580.2004.33903.x

14. Gerreth K.; Gerreth P. Occurrence of oral trauma in young epileptic patients. Eur J Paediatr Dent. 2014, 15 (1): 13-6.

h t t p : / / admin.ejpd.eu/download / EJPD_2014_1_2.pdf

15. Costa ALF, Yasuda CL, França MC, Morita ME, Cendes F. Refractory epilepsy is highly associated with severe dentoalveolar and maxillofacial injuries. Epileptic Disord. 2011, 13 (1): 61-4.

https://link.springer.com/article/10.1684/ epd.2011.0401

16. Ogunbodede EO, Adamolekun B., Akintomide AO. Oral health and dental treatment needs in Nigerian patients with epilepsy. Epilepsia. 1998, 39 (6): 590-4.

https://onlinelibrary.wiley.com/doi/ pdf/10.1111/j.1528-1157.1998.tb01426.x

17. Kirby S., Sadler RM. Injury and Death as a Result of Seizures. Epilepsia. 1995, 36 (1): 25-8.

https://onlinelibrary.wiley.com/doi/ abs/10.1111/j.1528-1157.1995.tb01660.x

18. Kumar P., Bhojraj N. Successful prevention of oral self-mutilation using a lip guard: a case report. Spec Care Dentist. 2011, 31 (3): 114-8. 
https://onlinelibrary.wiley.com/doi/abs/10.1111/ j.1754-4505.2011.00188.x

19. Buck D., Baker GA, Jacoby A., Smith DF, Chadwick DW. Patients' experiences of injury as a result of epilepsy. Epilepsia. 1997, 38 (4): 439-44.

20. Mattson RH, Gidal BE. Fractures, epilepsy, and antiepileptic drugs. Epilepsy Behav. 2004, 5 (2): S36-40. https://doi.org/10.1111/j.1528-1157.1997. tb01733.x

\section{Tiamkao S., Sawanyawisuth K.} Asawavichienjinda T., Yaudnopakao P., Arunpongpaisal S., Phuttharak W. et al. Predictive risk factors of seizure-related injury in persons with epilepsy. J Neurol Sci. 2009, 285 (1-2): 59-61.

https://doi.org/10.1016/j.jns.2009.05.021

\section{Autor correspondente:}

Bruna Luiza Roim Varotto

\section{Endereço para correspondência:}

Instituto de PsiquiatriaHospital das Clínicas da Faculdade de Medicina da Universidade de São Paulo

R. Dr. Ovídio Pires de Campos, 875 - Cerqueira César

São Paulo - SP, CEP: 05403-903

Submetido em: 17-8-2018

Aceito em: 4-12-2018 